\title{
BMJ Open Systematic review of adherence rates by medication class in type 2 diabetes: a study protocol
}

\author{
Andrew McGovern, Zayd Tippu, William Hinton, Neil Munro, Martin Whyte, \\ Simon de Lusignan
}

To cite: McGovern A, Tippu Z, Hinton W, et al. Systematic review of adherence rates by medication class in type 2 diabetes: a study protocol. BMJ Open 2016;6:e010469. doi:10.1136/bmjopen-2015010469

- Prepublication history and additional material is available. To view please visit the journal (http://dx.doi.org/ 10.1136/bmjopen-2015010469).

Received 6 November 2015 Revised 1 February 2016 Accepted 10 February 2016

CrossMark

Section of Clinical Medicine and Aging, University of Surrey, Guildford, UK

Correspondence to Dr Andrew McGovern; a.mcgovern@surrey.ac.uk

\section{ABSTRACT}

Introduction: Treatment options for type 2 diabetes are becoming increasingly complex with people often prescribed multiple medications, and may include both oral and injectable therapies. There is ongoing debate about which drug classes provide the optimum second-line and third-line treatment options. In the real world, patient adherence and persistence determines medication effectiveness. A better understanding of adherence may help inform the choice of second-line and third-line drug classes.

Methods and analysis: This systematic review will compare adherence and persistence rates across the different classes of medication available to people with type 2 diabetes. It will include all identified studies comparing medication adherence or persistence between two or more glucose-lowering medications in people with type 2 diabetes. Research databases (MEDLINE, EMBASE, The Cochrane Library, The Register of Controlled Trials, PsychINFO and CINAHL) will be searched for relevant articles, using a comprehensive search strategy. All identified medication trials and observational studies will be included which compare adherence or persistence across classes of diabetes medication. The characteristics and outcomes of all the included studies will be reported along with a study quality grade, assessed using the Cochrane Risk Assessment Tool. The quality of adjustment for confounders of adherence or persistence will be reported for each study. Where multiple $(n \geq 3)$ studies provide compare adherence or persistence across the same 2 medication classes, a meta-analysis will be performed.

Ethics and dissemination: No ethics approval is required. This review and meta-analysis (where possible) will provide important information on the relative patient adherence and persistence, with the different classes of diabetes therapies. Once complete, the results will be made available by peer-reviewed publication.

Trial registration number: CRD42015027865.

\section{INTRODUCTION}

Type 2 diabetes is a growing problem worldwide. $^{1}$ Alongside this increased prevalence comes an increased burden of disease from complications, which are potentially preventable. ${ }^{2}$ The importance of patient education and promoting self-care has long been recognised as a key component in chronic disease management and improving patient outcomes. ${ }^{3}$ One component of self-care is adherence to often complicated medication regimes. Good adherence is associated with reduced risk of diabetes complications, reduced mortality and reduced economic burden. ${ }^{4-6}$ However, a substantial proportion of people with type 2 diabetes do not take medication as prescribed, with only $67-85 \%$ of oral medication doses taken, and approximately $60 \%$ of insulin doses. ${ }^{7}$

In recent years, new medication classes have been introduced which have increased the complexity of diabetes treatment algorithms leading to multiple second-line and third-line options. ${ }^{8-10}$ Selection from these options usually follows a discussion with the patient and involves assessing potential efficacy, contraindications, potential side effects (especially hypoglycaemia risk and weight change), medication cost, frequency of dosing, whether the medication is required to be administered as an injection and whether monitoring of blood indices is required. However, a key component of medication effectiveness, rarely discussed at the outset, is patient adherence, and this is especially important in diabetes as overall adherence to therapies is poor.

Adherence is determined by many factors: patient-related, condition-related, socioeconomic, health system-related and therapy-related (table 1) factors. ${ }^{11}$ While interventions aimed at improving medication adherence should target all these main domains, it is the therapy-related component that will predominantly influence which therapy is chosen. Identification of any substantial differences in adherence rates 
Table 1 Factors previously identified as associated with medication adherence in people with diabetes categorised by the five dimensions affecting adherence as defined by $\mathrm{WHO}^{11}$

\begin{tabular}{|c|c|}
\hline Dimension & $\begin{array}{l}\text { Factors associated with reduced } \\
\text { adherence }\end{array}$ \\
\hline Patient-related & $\begin{array}{l}\text { Younger age, }{ }^{5}{ }^{26-28} \text { female } \\
\text { gender, }{ }^{26}{ }^{27} \text { smoking, }^{5} \text { impulsivity, } \\
\text { ethnic minority groups }\end{array}$ \\
\hline Socioeconomic & $\begin{array}{l}\text { Lower socioeconomic status, }{ }^{28}{ }^{32} \text { lack } \\
\text { of financial resources/increased } \\
\text { medication costs, }{ }^{26} 33{ }^{34} \text { lower } \\
\text { education level, }{ }^{27}{ }^{35} \text { lack of family } \\
\text { support }^{36}\end{array}$ \\
\hline Condition-related & $\begin{array}{l}\text { Presence of depression, }{ }^{37} \text { or other } \\
\text { chronic diseases, }{ }^{38-40} \text { shorter duration } \\
\text { of diabetes, }{ }^{24} \text { fewer diabetes } \\
\text { complications }{ }^{28}\end{array}$ \\
\hline Health system & $\begin{array}{l}\text { Healthcare centre/clinic, }{ }^{41} \text { increased } \\
\text { distance to nearest pharmacy, }{ }^{42} \text { lower } \\
\text { continuity of care }{ }^{43}\end{array}$ \\
\hline Therapy-related & $\begin{array}{l}\text { Concurrent medication use, }{ }^{25} 384445 \\
\text { adverse effects, }{ }^{46} 47 \text { poor previous } \\
\text { experience with medication }{ }^{42}\end{array}$ \\
\hline
\end{tabular}

between medication classes is vitally important. While there have been previous reviews of medication adherence in type 2 diabetes, these have not specifically interrogated differences in adherence rates between medications. ${ }^{7} 12-16$ The side-effect profile of each class of diabetes medication is different, and is likely to have a differential impact on adherence. Similarly, different dosing regimens and administration routes between classes will influence adherence. Knowledge of any differential in adherence rates between classes will help to inform prescribing practice, and is currently lacking.

A wide range of terms have been used to describe patient use (or non-use) of prescribed medications including: adherence, compliance, concordance and persistence. Two distinct patterns of medication use are described by these terms; missed medication doses (generally described by the terms adherence, compliance and concordance) and duration of use before termination (persistence). The term concordance is preferred by some to emphasise the joint agreement by the prescribing physician and the patient to use the prescribed medication in a certain way. ${ }^{17}$ 'Adherence' is the term recommended for use by $\mathrm{WHO}^{18}$ however, the term most commonly used in the literature is compliance. ${ }^{19}$ For this study, we will use the term adherence. Persistence with medication is the preferred term to refer to the duration of medication use. ${ }^{19}$ This is another key component of effectiveness of clinical medication, and is closely linked with medication adherence.

Knowing the relative adherence and persistence rates of medications used for the treatment of type 2 diabetes is important when selecting from the increasingly complex array of therapies.

\section{METHODS}

\section{Research objective}

This systematic review will compare adherence and persistence rates between different classes of therapies for the treatment of hyperglycaemia in people with type 2 diabetes. It will attempt to answer the question: Are there substantially different adherence and persistence rates between the different classes of therapies available for type 2 diabetes? The key components of the study are outlined in table 2.

Literature that compares adherence rates and persistence between different medication classes will be identified and, where appropriate, synthesised and summarised. This review forms an early component of a wider research theme: the University of Surrey-Lilly Real World Evidence projects. These projects aim to use routine primary care records to identify areas for improvement of diabetes management. This review will inform further research as part of these projects, and by the wider research community into medication adherence.

\section{Interventions}

The adherence and persistence with all available glucose-lowering therapies will be explored. These will be grouped by drug class for comparison (table 3). We will include medications which are in current use, those which have been previously withdrawn or discontinued, and those which have not yet been licensed, if sufficient data on adherence is available. Data on medications which are not currently in general use may provide important information on the adherence to the medication class, for example, insulin inhalation (trade name, Exubera) - an inhaled insulin discontinued in 2007may provide useful information on adherence to inhaled insulin use.

We will perform secondary analyses to identify differences in adherence and persistence within classes where daily, weekly and depot preparations exist (such as glucagone-like peptide 1 agonists). We will also perform a secondary analysis of the difference in adherence and persistence with the biguanide metformin standard

Table 2 The key components of the systematic review described using the standard PICOS (Participants, Interventions, Comparisons, Outcomes and Study design) formula

\begin{tabular}{ll}
\hline Participants & $\begin{array}{l}\text { People with type 2 diabetes who required } \\
\text { treatment with one or more medications } \\
\text { Interventions }\end{array}$ \\
Glucose-lowering medications \\
Other classes of glucose-lowering \\
medications (metformin to be used as the \\
default comparator where available) \\
Study design & $\begin{array}{l}\text { Any reported measure of patient adherence } \\
\text { or persistence with treatment } \\
\text { Both interventional and observational } \\
\text { studies }\end{array}$ \\
\hline
\end{tabular}


Table 3 Classes of medications used in type 2 diabetes

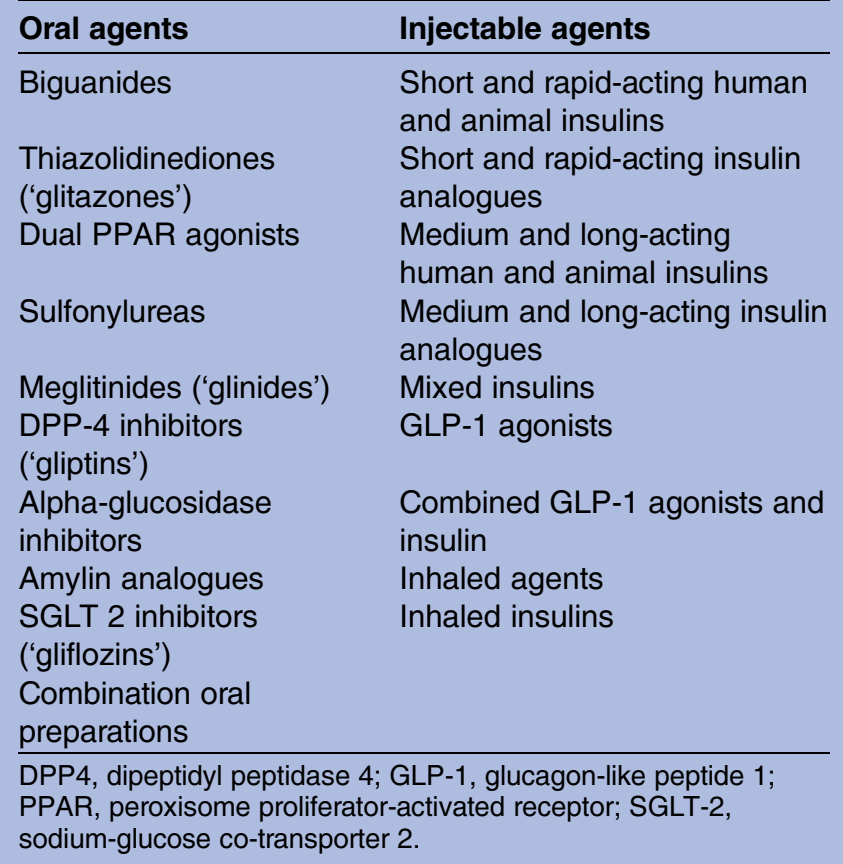

formulation and metformin-modified release. In our experience, there is a substantial difference in tolerability of these preparations.

\section{Comparisons}

Medication classes will be compared (within individual studies) with other available medication classes. Given the previously identified ${ }^{7}$ heterogeneity in studies designs, and both measures and reporting methods of adherence, it is likely that direct comparison of classes will have to be intrastudy only. For this reason, studies reporting adherence or persistence rates in a single drug class will be excluded.

\section{Outcome measures}

Adherence and persistence will be defined as:

- Adherence: The proportion (or estimated proportion) of doses taken over a defined time, or the proportion of people attaining a defined level of adherence. Where possible, we will use the recommended definition of adequate adherence to be a medication possession ratio (MPR) $\geq 80 \%$ or proportion of days covered (PDC) $\geq 80 \%^{20}$ or proportion of doses taken (when measured directly) to be $\geq 80 \%$. MPR is defined as the number of days' supply of medication divided by the medication refill interval (with some variation in the formula used). PDC is defined as proportion of days the patient has medication available in a defined period. PDC allows adjustment for overlaps caused by early collection of prescriptions.

- Persistence. The proportion of people who remain on a therapy after a defined period of time, or the mean duration of therapy. Persistence may be defined as medication refills consistent with ongoing use of the medication. ${ }^{20}$ Failure to refill a prescription after a predefined gap indicates non-persistence.

Differing definitions of adherence affect outcomes, with the impact of this effect apparent when stratifying studies by the definition used. ${ }^{21}$ We will duplicate this method; stratifying studies by the threshold used to define adequate adherence, and use a similar method to stratify studies by the definition of persistence (see Data synthesis section).

Primary outcomes

- Comparison of adherence or persistence between metformin (standard formulation) and other hypoglycaemic agents.

Secondary outcomes

- Comparison of adherence or persistence between two or more hypoglycaemic agents, where metformin is not the comparator.

- Any differences between interventional and observational reports of studies.

- Analysis of the impact of the definition of adherence/persistence on the relative adherence rates to diabetes therapies.

- Any reported reasons for differences in adherence or persistence between medication classes.

- Any reported differences in disease outcomes (glycaemic control or diabetes complications) in patients due to differing adherence rates between medication classes.

- Assessment of the quality of adjustment for confounding in existing studies.

\section{Included study types}

All interventional and observational studies comparing adherence rates and/or persistence with two or more diabetes medication classes will be included. Interventional studies types will include randomised, non-randomised, and cross-over trials. Interventions can include interventions aimed at improving adherence or the use of medication as the intervention (clinical trials). Observational studies will include cohort and cross-sectional studies (eg, those with surveys of patient reported compliance).

\section{Study inclusion criteria}

Studies will be included in the review if all the participants taking the medications of interest have type 2 diabetes. Studies from the community setting will be included; hospital outpatient studies and community studies, including primary care. Studies measuring adherence using the following methods will be included; self-reported measures, clinician estimated adherence, calculated adherence rates from prescribing or dispensing data, or electronic monitoring of medication use. Electronic monitoring technology broadly involves the use of a medication container which collects data on bottle opening.

Studies will be included if they report a sufficient measure of adherence and/or persistence. For this purpose, adherence will be defined as the proportion of 
doses taken over a given time period (period defined in the study); reported as a fraction; or reported as a proportion of patients achieving a predefined level of adherence. Persistence will be defined as the proportion of people who remain taking a therapy after a specified treatment period or the mean number of days until discontinuation. These definitions are consistent with previous work. ${ }^{7}$

\section{Exclusion criteria}

Studies will be excluded if

- They include populations with only type 1 diabetes, people without diabetes taking the medication of interest, or mixed populations where adherence rates in those with type 2 diabetes were not reported separately.

- The medications studied are not used to treat diabetes.

- No clear measure of adherence or persistence is reported.

- Adherence or persistence rates in diabetes during pregnancy.

- Adherence or persistence rates with medications from a single class only are reported.

- Adherence or persistence rates are not reported separately by individual drug or by drug class.

- The study participants are hospital inpatients or inpatients at another institution.

- The study participants have medication administered by a caregiver or professional.

\section{Search method}

Studies will be obtained by searching the following databases:

- MEDLINE

- EMBASE

- The Cochrane Library including the Cochrane Controlled Trials Register (CENTRAL) and the Database of reviews of effectiveness (DARE) and the NHS Health Economics Database

- The meta Register of Controlled Trials (http://www. controlled-trials.com)

- PsychINFO

- CINAHL

Additionally, bibliographic searches of the included studies will also be performed. Only studies published from 2006 onwards will be included to provide a summary of the most recent 10 years of publications.

\section{Search terms}

The search strategy detailed in online supplementary appendix 1 will be used to search the MEDLINE database. This strategy will be adapted to apply to the other included databases. Only English language studies will be included.

\section{Study selection}

Two clinicians will independently review the titles and abstracts of all the articles identified using the search terms described. Studies will be included which match the above inclusion criteria and do not have any exclusion characteristics. Studies will be eliminated if both reviewers agreed that the study did not meet the required criteria. Studies which either or both reviewers feel merit further analysis will be included for full paper review. Full articles will be collected and collated into unique studies. Full papers for each study will be reviewed separately by each reviewer against the inclusion and exclusion criteria. In the case of disagreement between the reviewers, a third reviewer will be included to resolve the discrepancy. If amendments are needed to the inclusion or exclusion criteria to clarify whether a particular study is included or excluded these will be reported in the final review manuscript alongside details of the paper(s) in question.

\section{Data extraction}

Data will be extracted using a standardised method into a preformatted database. Data will be extracted on study type and intervention (if relevant), study location, sample size, study population characteristics (age, gender, comorbidities, concurrent medication use-including pill burden, and duration of diabetes), duration of follow-up, inclusion and exclusion criteria, and funding sources. Outcome data extracted will comprise adherence or persistence measurement method, adherence values, persistence values, suggested or measured reasons for any reported interclass adherence or persistence differences, and impact on glucose control and diabetes complications. All extracted data will be verified by a second reviewer.

\section{Assessment of evidence quality}

The internal validity of all included studies will be assessed using the Cochrane risk assessment tools. This enables evaluation of clinical trials based on the following criteria; sequence generation, allocation concealment, blinding of participants, personnel and outcome assessors, incomplete outcome data, selective outcome reporting, and other sources of bias. All publications will be categorised as either low risk, unclear risk or high risk or bias. For cohort studies, the risk assessment tool enables assessment based on the following domains; selection of population, assessment of exposure, assessment of the present of outcomes at baseline (not relevant here), matching or adjustment for confounders, identification of prognostic variables, assessment of the outcome measure, adequacy of follow-up, and similarity of co-interventions between groups. We will report the quality of all the included studies, and will comment on the overall impact of data quality on the conclusions drawn by the review. We will rank the overall quality of evidence on this topic using the GRADE (Grading of Recommendations Assessment, Development and Evaluation) scoring system. ${ }^{22}$

\section{Data analysis and presentation}

We will provide a description of the number of studies included and excluded at each stage of the search 
process. A study flow diagram will be incorporated in the standard form suggested by the Preferred Reporting Items for Systematic reviews and Meta-Analyses (PRISMA) statement. ${ }^{23}$ The characteristics of all the studies included will be presented with the study outcome findings. We will provide a text summary of the main results, and a discussion including details of the quality of existing evidence and recommendations for future research.

\section{Data synthesis}

Factors such as age, gender, comorbidities and concurrent medication use influence both medication choice and adherence. ${ }^{5} 2425$ Untangling the contribution to non-adherence caused by these confounders from that caused by the medication class will be complex. We will address this issue in several ways:

1. We will perform a comparison of adherence between classes in randomised trials. Where randomisation is sufficient, any differences in adherence are likely to be related to medication factors only.

2. In observational studies, we will assess and report the methods used to identify and adjust for potential confounding factors (table 1). We will report which confounders have been adjusted for in each included study, and report those which have not been measured or reported. We will comment on the limitation of the methods used to control for confounders where this has been performed.
3. We compare relative adherence rates for each drug comparison in all studies (via meta-analysis as described below) with those with a method of adjustment or controlling for confounders. These methods may include adjustment via statistical methods, such as regression analysis, or by controlling for confounding via case selection (such as case-control studies).

4. We will perform meta-analyses for each pair of medication classes compared where there are a sufficient number of studies enabling comparison $(n \geq 3)$. The possible pairwise comparisons are shown in figure 1 . For each pairwise comparison, we will undertake the following analyses: a comparison of the proportion of people adherent to medication all studies (stratified by the definition used for adherence; all definitions, $\geq 75 \%$ of doses taken/MPR/PDC, $\geq 80 \%$ doses taken/MPR/PDC, $\geq 90 \%$ doses taken/MPR/PDC).

5. A comparison of the proportion of people adherent to medication stratified by study design; interventional studies, all observational studies, and only observational studies which adjust for confounders of adherence.

6. A comparison of persistence duration in all studies (stratified by the definition used for persistence; all definitions providing a measure of duration, nonpersistence gap between refills $\geq 90$ days, nonpersistence gap between refills $\geq 60$ days).

7. A comparison of persistence duration stratified by study design; interventional studies, all observational

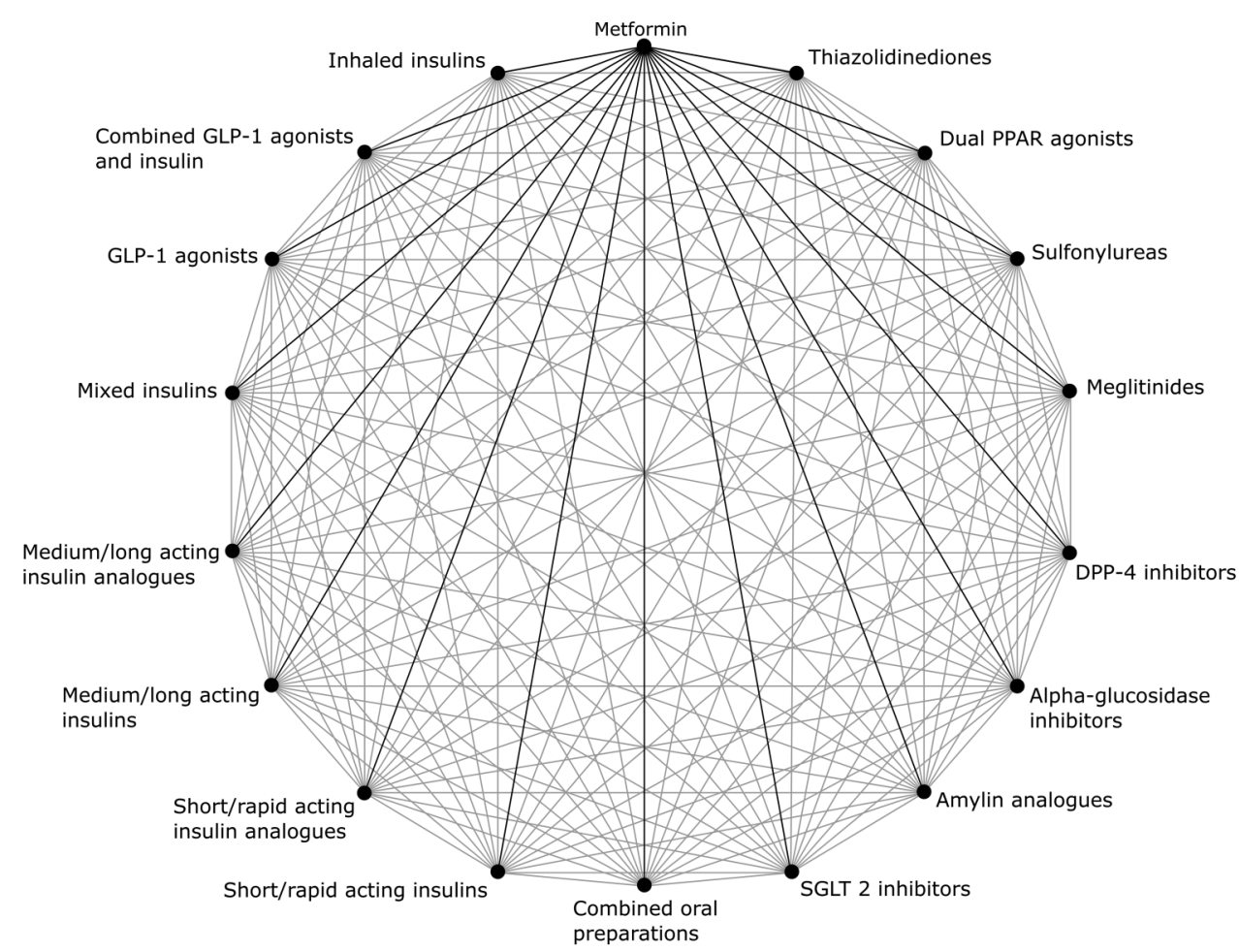

Figure 1 A schematic representation of the possible pairwise comparisons of adherence and persistence between different medication classes. Comparison with metformin is the primary outcome (black lines), but the additional pairwise comparisons possible are secondary outcomes of interest (grey lines). 
studies, and only observational studies which adjust for confounders of persistence.

For all meta-analyses, we will report tests for heterogeneity ( $Q$ statistics and $\mathrm{I}^{2}$ ).

\section{Review registration}

This review has been registered with PROSPERO (registration: CRD42015027865).

\section{Updates to study protocol}

If any updates to the study protocol are required, these will be listed and included as supplementary information alongside a final manuscript, and updated on the PROSPERO register.

\section{Review time frames}

We will aim to complete the review within a period of 1 year from the start of the literature search. This time period excludes time spent following submission of a final manuscript and article peer review.

\section{CONCLUSIONS}

This review and, if possible, meta-analysis, will provide important information on patient adherence and persistence with the different classes of diabetes therapies. This is one of the key components of medication effectiveness, and will help to guide clinical decision-making about which is the optimum second-line or third-line diabetes therapy to initiate. The review will also enable key recommendation for further research in this area to be made.

Contributors AM wrote the manuscript and designed the review. ZT, WH, $\mathrm{NM}, \mathrm{MW}$, and SdL critically appraised the review design and systematic review proposal manuscript. All authors have approved the final manuscript.

\section{Funding Eli Lilly and Company.}

Competing interests AMG, WH, and SdL are funded as part of the University of Surrey-Lilly Real World Evidence (RWE) projects. This centre is funded by Eli-Lilly Pharmaceuticals. Lilly are updated periodically with the research performed as part of the RWE projects although these funders have not had a role in development of this protocol. NM has received fees for serving as a speaker, a consultant or an advisory board member for Allergan,

Bristol-Myers Squibb-Astra-Zeneca, GlaxoSmithKline, Eli Lilly, Lifescan, MSD, Metronic, Novartis, Novo Nordisk, Pfizer, Sankio, Sanofi, Roche, Servier, Takeda. MW has received speaker fees from Astra-Zeneca.

Provenance and peer review Not commissioned; externally peer reviewed.

Open Access This is an Open Access article distributed in accordance with the Creative Commons Attribution Non Commercial (CC BY-NC 4.0) license, which permits others to distribute, remix, adapt, build upon this work noncommercially, and license their derivative works on different terms, provided the original work is properly cited and the use is non-commercial. See: http:// creativecommons.org/licenses/by-nc/4.0/

\section{REFERENCES}

1. Chen L, Magliano DJ, Zimmet PZ. The worldwide epidemiology of type 2 diabetes mellitus-present and future perspectives. Nat Rev Endocrinol 2011;8:228-36.

2. Hu FB. Globalization of diabetes: the role of diet, lifestyle, and genes. Diabetes Care 2011;34:1249-57.
3. Norris SL, Engelgau MM, Narayan KM. Effectiveness of self-management training in type 2 diabetes: a systematic review of randomized controlled trials. Diabetes Care 2001;24:561-87.

4. Ho PM, Bryson CL, Rumsfeld JS. Medication adherence: its importance in cardiovascular outcomes. Circulation 2009;119:3028-35.

5. Currie CJ, Peyrot M, Morgan CL, et al. The impact of treatment noncompliance on mortality in people with type 2 diabetes. Diabetes Care 2012;35:1279-84.

6. Muszbek N, Brixner D, Benedict A, et al. The economic consequences of noncompliance in cardiovascular disease and related conditions: a literature review. Int J Clin Pract 2008:62:338-51.

7. Cramer JA. A systematic review of adherence with medications for diabetes. Diabetes Care 2004;27:1218-24.

8. National Institute for Health and Clinical Excellence. NICE guidelines [CG66]-Type 2 diabetes, 2008. https://www.nice.org.uk/guidance/cg66

9. National Institute for Health and Clinical Excellence. NICE guidelines [CG87]-Type 2 diabetes: The management of type 2 diabetes, 2009. https://www.nice.org.uk/guidance/cg87

10. National Institute for Health and Clinical Excellence. Dapagliflozin in combination therapy for treating type 2 diabetes: NICE technology appraisals [TA288], 2013. http://www.nice.org.uk/Guidance/ta288

11. Sabaté E. Adherence to Long-Term Therapies-Evidence for Action 2003. whqlibdoc.who.int/publications/2003/9241545992.pdf

12. Asche C, LaFleur J, Conner C. A review of diabetes treatment adherence and the association with clinical and economic outcomes. Clin Ther 2011;33:74-109.

13. Breitscheidel L, Stamenitis S, Dippel FW, et al. Economic impact of compliance to treatment with antidiabetes medication in type 2 diabetes mellitus: a review paper. J Med Econ 2010;13:8-15.

14. Vermeire E, Wens J, Van Royen P, et al. Interventions for improving adherence to treatment recommendations in people with type 2 diabetes mellitus. Cochrane Database Syst Rev 2005;(2): CD003638.

15. Bailey CJ, Kodack M. Patient adherence to medication requirements for therapy of type 2 diabetes. Int J Clin Pract 2011;65:314-22.

16. Rubin RR. Adherence to pharmacologic therapy in patients with type 2 diabetes mellitus. Am J Med 2005;118(Suppl 5A):27S-34S.

17. Aronson JK. Compliance, concordance, adherence. Br J Clin Pharmacol 2007;63:383-4.

18. World Health Organisation. Adherance to long-term therapies: Evidence for action, 2003. http://www.who.int/chp/knowledge/ publications/adherence full report.pdf

19. Cramer JA, Roy A, Burrell A, et al. Medication compliance and persistence: terminology and definitions. Value Health 2008;11:44-7.

20. Raebel MA, Schmittdiel J, Karter AJ, et al. Standardizing terminology and definitions of medication adherence and persistence in research employing electronic databases. Med Care 2013;51(8 Suppl 3):S11-21.

21. Simpson SH, Eurich DT, Majumdar SR, et al. A meta-analysis of the association between adherence to drug therapy and mortality. BMJ 2006;333:15.

22. GRADE Working Group. GRADE (Grading of Recommendations Assessment, Development and Evaluation). 2015. http://www. gradeworkinggroup.org/

23. Moher D, Liberati A, Tetzlaff J, et al. Preferred reporting items for systematic reviews and meta-analyses: the PRISMA statement. Ann Intern Med. 2009;151:264-9, W64.

24. Kirkman MS, Rowan-Martin MT, Levin R, et al. Determinants of adherence to diabetes medications: findings from a large pharmacy claims database. Diabetes Care 2015;38:604-9.

25. Lloyd CE, Mughal S, Roy T, et al. What factors influence concordance with medications? Findings from the UK Asian Diabetes study. Diabet Med 2014;31:1600-9.

26. Awodele O, Osuolale JA. Medication adherence in type 2 diabetes patients: Study of patients in Alimosho general hospital, Igando, Lagos, Nigeria. Afr Health Sci 2015;15:513-22.

27. Fisher L, Polonsky WH, Schikman $\mathrm{CH}$, et al. Emotional distress is linked to medication adherence in type 2 diabetes. Diabetes 2009;58:A478.

28. Bezie $\mathrm{Y}$, Molina $\mathrm{M}$, Hernandez $\mathrm{N}$, et al. Therapeutic compliance: a prospective analysis of various factors involved in the adherence rate in type 2 diabetes. Diabetes Metab 2006;32:611-16.

29. Reach $\mathrm{G}$. Two character traits associated with adherence to long term therapies. Diabetes Res Clin Pract 2012;98:19-25.

30. Egede LE, Gebregziabher M, Hunt KJ, et al. Regional, Geographic, and Ethnic Differences in Medication Adherence Among Adults with Type 2 Diabetes. Ann Pharmacother 2011;45:169-78. 
31. Shenolikar RA, Balkrishnan R, Camacho FT, et al. Race and medication adherence in medicaid enrollees with type-2 diabetes. J Natl Med Assoc 2006;98:1071-7.

32. Guénette L, Moisan J, Breton MC, et al. Difficulty adhering to antidiabetic treatment: factors associated with persistence and compliance. Diabetes Metab 2013;39:250.

33. Parveen M, Piyarali S. Treatment compliance to diabetes care: across-sectional study from Pakistan. Value Health 2011;14: A98-A9.

34. Gibson TB, Song X, Alemayehu B, et al. Cost sharing, adherence, and health outcomes in patients with diabetes. Am J Manag Care 2010;16:589-600.

35. Khowaja LA. Treatment compliance to diabetes care: across-sectional study from Pakistan. J Diabetes Sci Technol 2012;6:A75.

36. Tiv M, Viel JF, Mauny F, et al. Medication adherence in type 2 diabetes: the ENTRED study 2007, a French population-based study. PLOS ONE 2012;7:e32412.

37. Gonzalez JS, Peyrot M, McCarl LA, et al. Depression and diabetes treatment nonadherence: a meta-analysis. Diabetes Care 2008;31:2398-404

38. Leblond J, Pilon D, Beaudette C, et al. Predictors of nonpersistence with thiazolidinediones in patients with type 2 diabetes. Can $J$ Diabetes 2005;29:95-102.

39. Gwadry-Sridhar F, Leslie RS, Patel B, et al. Influence of previous medication compliance on future compliance in patients with type 2 diabetes. Diabetes 2010;59:A54.
40. Toyoda M, Yokoyama $\mathrm{H}, \mathrm{Abe} \mathrm{K}$, et al. Predictors of response to liraglutide in Japanese type 2 diabetes. Diabetes Res Clin Pract 2014; $106: 451$

41. Bryson $\mathrm{CL}, \mathrm{Au} \mathrm{DH}$, Maciejewski $\mathrm{ML}$, et al. Wide clinic-level variation in adherence to oral diabetes medications in the VA. J Gen Intern Med 2013;28:698-705.

42. Gwadry-Sridhar F, Leslie RS, Patel B, et al. Understanding Predictors of Compliance in Fixed-Dose Combination vs LooseDose Combination Therapy for Treatment of Type 2 Diabetes. Diabetes 2010;59:A346.

43. Chen $\mathrm{CC}$, Tseng $\mathrm{CH}$, Cheng SH. Continuity of care, medication adherence, and health care outcomes among patients with newly diagnosed type 2 diabetes: a longitudinal analysis. Med Care 2013;51:231-7.

44. Gravenstein S, Thompson SF, Stefanacci RG. Diabetes Treatment Patterns and Adherence among Elderly Patients from a US Managed Care Claims Database. Diabetes 2009;58:A518.

45. Donnan PT, MacDonald TM, Morris AD. Adherence to prescribed oral hypoglycaemic medication in a population of patients with Type 2 diabetes: a retrospective cohort study. Diabet Med 2002;19:279-84.

46. Laires P, Iglay K, Fan CPS, et al. Impact of hypoglycemia on discontinuing or down-titrating sulfonylurea among type 2 diabetes patients without insulin. Value Health 2014;17:A353.

47. Grant RW, Devita NG, Singer DE, et al. Polypharmacy and medication adherence in patients with type 2 diabetes. Diabetes Care. 2003;26:1408-12. 